\title{
HIGHER DIMENSIONAL KNOTS IN TUBES
}

\author{
BY \\ YAICHI SHINOHARA( $\left.{ }^{(}\right)$
}

\begin{abstract}
Let $K$ be an $n$-knot in the $(n+2)$-sphere and $V$ a tubular neighborhood of $K$. Let $L^{\prime}$ be an $n$-knot contained in a tubular neighborhood $V^{\prime}$ of a trivial $n$-knot and $L$ the image of $L^{\prime}$ under an orientation preserving diffeomorphism of $V^{\prime}$ onto $V$. The purpose of this paper is to show that the higher dimensional Alexander polynomial and the signature of the $n$-knot $L$ are determined by those of $K$ and $L^{\prime}$.
\end{abstract}

1. Introduction. An $n$-knot $K$ is a smooth oriented submanifold of the oriented $(n+2)$-sphere $S^{n+2}$ which is homeomorphic to $S^{n}$. Throughout the paper we assume that the orientation of $S^{n+2}$ is fixed. Two $n$-knots $K_{1}$ and $K_{2}$ are said to be of the same $n$-knot type if there exists an orientation preserving homeomorphism $f$ of $S^{n+2}$ onto itself such that $f\left(K_{1}\right)=K_{2}$ and $f \mid K_{1}$ is orientation preserving. By a tube (or an open tube) of an $n$-knot $K$ we mean a closed (or an open) tubular neighborhood of $K$ in $S^{n+2}$.

Let $K$ be an $n$-knot in $S^{n+2}$ and $V$ a tube of $K$. Let $V^{\prime}$ be a tube of a trivial $n$-knot $K^{\prime}$. Let $f: V^{\prime} \rightarrow V$ be a diffeomorphism of $V^{\prime}$ onto $V$ which preserves the orientations induced by $S^{n+2}$ in $V^{\prime}$ and $V$. If $n=1$, we further assume that $f$ transforms longitudes into longitudes. Let $L^{\prime}$ be an $n$-knot contained in the interior of $V^{\prime}$. Then $L^{\prime} \sim \lambda K^{\prime}$ in $V^{\prime}$ for some integer $\lambda$. Moreover, $L=f\left(L^{\prime}\right)$ is an $n$-knot contained in the interior of $V$ and $L \sim \lambda K$ in $V$.

For the case $n=1, \mathrm{H}$. Seifert [4] showed that

$$
\Delta_{L}(t)=\Delta_{K}\left(t^{\lambda}\right) \Delta_{L^{\prime}}(t),
$$

where $\Delta_{L}(t), \Delta_{K}(t)$ and $\Delta_{L^{\prime}}(t)$ are the Alexander polynomials of $L, K$ and $L^{\prime}$, and the author [5] proved that

$$
\begin{aligned}
& \sigma(L)=\sigma\left(L^{\prime}\right) \quad \text { when } \lambda \text { is even, } \\
& =\sigma(K)+\sigma\left(L^{\prime}\right) \text { when } \lambda \text { is odd, }
\end{aligned}
$$

where $\sigma(L), \sigma(K)$ and $\sigma\left(L^{\prime}\right)$ are the signatures of $L, K$ and $L^{\prime}$ defined by H. F. Trotter [6].

The purpose of this paper is to generalize these results to the case of higher dimensional knots.

Received by the editors May 18, 1970.

AMS 1969 subject classifications. Primary 5520; Secondary 5720.

Key words and phrases. $q$ th dimensional Alexander polynomial, signature of $n$-knots.

(1) The author is a postdoctoral fellow of the National Research Council of Canada (Grant A4034).

Copyright (C) 1971, American Mathematical Society 
Following J. W. Milnor [3], we will define the $q$ th dimensional Alexander polynomial $\Delta_{K}^{q}(t)$ of an $n$-knot $K$ (see $\left.\S 2\right)$. We prove that

THEOREM 1.1. For $1 \leqq q \leqq n$,

$$
\Delta_{L}^{q}(t)=\Delta_{K}^{q}\left(t^{\lambda}\right) \cdot \Delta_{L^{\prime}}^{q}(t)
$$

In [2], D. Erle defined the signature $\sigma_{2}$ of a $(2 m-1)$-knot which is equivalent to $\sigma$ for $m=1$ [1]. For the case when $m$ is even, another signature $\sigma_{1}$ of a $(2 m-1)$-knot was also defined. We show that

THEOREM 1.2. If $n=2 m-1$, then

$$
\begin{aligned}
\sigma_{2}(L) & =\sigma_{2}\left(L^{\prime}\right) & & \text { when } \lambda \text { is even, } \\
& =\sigma_{2}\left(L^{\prime}\right)+(-1)^{(m+1)(\lambda-1) / 2} \sigma_{2}(K) & & \text { when } \lambda \text { is odd. }
\end{aligned}
$$

Furthermore, if $m$ is even, then $\sigma_{1}(L)=\sigma_{1}\left(L^{\prime}\right)+\lambda \sigma_{1}(K)$.

2. Preliminaries. $Z$ and $Q$ will denote the ring of integers and the field of rational numbers respectively. Throughout the paper we assume that every covering space is connected.

Let $X$ be a connected, locally path-connected and semilocally 1-connected space and $Y$ a subspace of $X$ which is connected and locally path-connected. Suppose that there exists a homomorphism

$$
\varphi: \pi_{1}\left(X, y_{0}\right) \rightarrow G
$$

of $\pi_{1}\left(X, y_{0}\right)$ onto an abelian group $G$, where $y_{0}$ is a point of $Y$. Then there exists a regular covering space $p: \tilde{X} \rightarrow X$ which belongs to $\operatorname{Ker}(\varphi) . G$ acts on $\tilde{X}$ as the group of covering transformations. More precisely, one can describe the action of $G$ on $\tilde{X}$ as follows:

Choose a point $\tilde{y}_{0} \in p^{-1}\left(y_{0}\right)$. Let $g$ be an element of $G$ and $\gamma$ an element of $\pi_{1}\left(X, y_{0}\right)$ which is mapped into $g$ by $\varphi$. Then there exists a unique path class $\tilde{\gamma}$ in $\tilde{X}$ with the initial point $\tilde{y}_{0}$ such that $p_{\#}(\tilde{\gamma})=\gamma$. The terminal point of $\tilde{\gamma}$ does not depend on the choice of $\gamma$ which satisfies $\varphi(\gamma)=g$. Hence $g$ corresponds to a unique covering transformation which maps $\tilde{y}_{0}$ into the terminal point of $\tilde{\gamma}$. This correspondence is an isomorphism of $G$ onto the group of covering transformations and does not depend on the choice of $\tilde{y}_{0} \in p^{-1}\left(y_{0}\right)$ (since $G$ is abelian).

The above described action of $G$ on $\tilde{X}$ is called the $\varphi$-action of $G$ on $\tilde{X}$. For every subgroup $S$ of $G$, the $\varphi$-action of $G$ naturally induces an action of $S$ on $\tilde{X}$.

Now we want to consider $p^{-1}(Y)$. Let $H$ be the image of $\pi_{1}\left(Y, y_{0}\right)$ under the homomorphism

$$
\psi=\varphi \cdot i_{\#}: \pi_{1}\left(Y, y_{0}\right) \rightarrow G
$$

where $i: Y \subset X$. Let $\left\{g_{1}=1, g_{2}, \ldots, g_{\mu}\right\}$ be a coset representative system of $G \bmod H$; that is, $G=H+g_{2} H+\cdots+g_{\mu} H$, where $\mu=[G: H]$ may not be finite. Let $Y_{1}$ be a path-component of $p^{-1}(Y)$ and $Y_{i}=g_{i}\left(Y_{1}\right)$ for $i=1,2, \ldots, \mu$. Then we have the following lemma. 
Lemma 2.1. (1) $p \mid Y_{i}: Y_{i} \rightarrow Y$ is a covering space which belongs to $\operatorname{Ker}(\psi)$, $i=1,2, \ldots, \mu$.

(2) For $i=1,2, \ldots, \mu$, the $\varphi$-action of $H$ on $\tilde{X}$ induces an action of $H$ on $Y_{i}$ by restricting every element of $H$ to $Y_{i}$. Furthermore, this induced action of $H$ on $Y_{i}$ coincides with the $\psi$-action of $H$ on $Y_{i}$.

(3) $p^{-1}(Y)$ is the disjoint union of $Y_{1}, Y_{2}, \ldots, Y_{\mu}$.

This lemma can be proved easily by using basic properties of covering spaces. Hence we omit the details.

Let $M$ be a finitely generated module over a principal ideal domain $P$. By the structure theorem for finitely generated modules over principal ideal domains, $M$ is isomorphic to a direct sum of cyclic modules; that is,

$$
M \cong P /\left(p_{1}\right) \oplus \cdots \oplus P /\left(p_{r}\right),
$$

where $\left(p_{i}\right)$ denotes the principal ideal spanned by an element $p_{i} \in P$. The generator of the product ideal $\left(p_{1} \cdots p_{r}\right)$, unique up to unit elements of $P$, will be called the order of $M$ and denoted by $\operatorname{order}_{P} M$ [3]. The order of $M$ is an invariant of the $P$-isomorphism class of $M$.

Now, in Lemma 2.1, suppose that $X$ is a finite connected simplicial complex, $Y$ a connected subcomplex of $X$ and $G=(t:)$ the infinite cyclic group generated by $t$. Then $H=\left(t^{\lambda}:\right)$ for some integer $\lambda$ and $p: \tilde{X} \rightarrow X$ is an infinite cyclic covering space. Let $\tilde{Y}$ denote $p^{-1}(Y)$. Since $\tilde{Y}$ is invariant under $t$ and $Y$ is a finite complex, $H_{*}(\tilde{Y} ; Q)$ is a finitely generated $\Gamma$-module, where $\Gamma$ denotes the rational group ring of $G$.

Suppose $\lambda \neq 0$. Then $[G: H]=|\lambda|=\mu$ and $\left\{1, t, \ldots, t^{\mu-1}\right\}$ forms a coset representative system of $G \bmod H$. Let $Y_{1}$ be a path-component of $\tilde{Y}$ and $Y_{i}=t^{i-1}\left(Y_{1}\right)$ for $i=1,2, \ldots, \mu$. By Lemma $2.1, p \mid Y_{i}: Y_{i} \rightarrow Y$ is an infinite cyclic covering space having $t^{\lambda}$ as a generator of the group of covering transformations. Hence $H_{*}\left(Y_{i} ; Q\right)$ is a finitely generated $\Gamma^{\lambda}$-module, where $\Gamma^{\lambda}$ is the rational group ring of $H$. Moreover, as $\Gamma^{\lambda}$-modules,

$$
H_{q}(\tilde{Y} ; Q) \cong H_{q}\left(Y_{1} ; Q\right) \oplus \cdots \oplus H_{q}\left(Y_{\mu} ; Q\right) .
$$

Since $t_{*}$ is an automorphism of $H_{q}(\tilde{Y} ; Q)$ which maps $H_{q}\left(Y_{1} ; Q\right)$ onto $H_{q}\left(Y_{2} ; Q\right)$, $\ldots, H_{q}\left(Y_{\mu} ; Q\right)$ onto $H_{q}\left(Y_{1} ; Q\right)$, a presentation of the $\Gamma^{\lambda}$-module $H_{q}\left(Y_{1} ; Q\right)$ can be considered as a presentation of the $\Gamma$-module $H_{q}(\tilde{Y} ; Q)$. This yields

Corollary 2.2. If $\Delta \in \Gamma^{\lambda}$ is the order of the $\Gamma^{\lambda}$-module $H_{q}\left(Y_{1} ; Q\right)$, then $\Delta$ is the order of the $\Gamma$-module $H_{q}(\tilde{Y} ; Q)$.

Suppose $\lambda=0$. Then $H$ is trivial and $\left\{t^{i}\right\}_{i \in Z}$ is a coset representative system for $G \bmod H$. Let $Y_{0}$ be a path-component of $\tilde{Y}$ and $Y_{i}=t^{i}\left(Y_{0}\right)$ for every integer $i$. By Lemma 2.1, $p \mid Y_{i}: Y_{i} \rightarrow Y$ is a homeomorphism and $\tilde{Y}$ is the disjoint union of $Y_{i}, i \in Z$. Hence we have 
CoRollary 2.3. If $\operatorname{dim}_{Q} H_{q}(Y ; Q)=\rho$, then $H_{q}(\tilde{Y} ; Q)$ is a free $\Gamma$-module of rank $\rho$.

Let $K$ be an $n$-knot in $S^{n+2}$ and $X$ the complement of an open tube $U$ of $K$ in $S^{n+2}$. Let $\varphi: \pi_{1}(X) \rightarrow G$ be a homomorphism of $\pi_{1}(X)$ onto an infinite cyclic group $G=(t:)$ defined by

$$
\varphi(g)=t^{\operatorname{Link}(g, K)} \quad \text { for } g \in \pi_{1}(X) .
$$

Then the covering space $p: \tilde{X} \rightarrow X$ belonging to $\operatorname{Ker}(\varphi)$ is an infinite cyclic covering space. $H_{q}(\tilde{X})$ is a finitely generated $\Lambda$-module, where $\Lambda$ is the integral group ring of $G$. Likewise $H_{q}(\tilde{X} ; Q) \cong H_{q}(\tilde{X}) \otimes_{z} Q$ is a finitely generated $\Gamma$-module. The order of the $\Gamma$-module $H_{q}(\tilde{X} ; Q)$ will be called the qth dimensional Alexander polynomial of $K$ and denoted by $\Delta_{K}^{q}(t)[3]$.

It follows from [2, p. 102] that

Proposition 2.4. The family $\left\{\Delta_{K}^{q}(t)\right\}_{q}$ is an invariant of the $n$-knot type of $K$.

Proposition 2.5. (1) $H_{q}(\tilde{X}) \stackrel{t-1}{\longrightarrow} H_{q}(\tilde{X})$ is a $\Lambda$-isomorphism for $q \neq 0$.

(2) $H_{q}(\tilde{X})\left(H_{q}(\tilde{X} ; Q)\right)$ is a torsion $\Lambda(\Gamma)$-module for every integer $q$.

(3) $H_{n+1}(\tilde{X}) \cong 0$.

(4) In the homology sequence of the pair $(\tilde{X}, \partial \tilde{X}), \partial_{*}: H_{n+1}(\tilde{X}, \partial \tilde{X}) \rightarrow H_{n}(\partial \tilde{X})$ is a $\Lambda$-isomorphism.

Proof. The proof of Assertion 5 in [3] holds for the integral homologies provided that $H_{*}(X) \cong H_{*}\left(S^{1}\right)$, from which (1) and (2) follow immediately.

Since there exists an $(n+1)$-dimensional subcomplex of $\tilde{X}$ which is a deformation retract of $\tilde{X}, H_{n+1}(\tilde{X})$ is a free $\Lambda$-module. Hence, by $(2), H_{n+1}(\tilde{X}) \cong 0$.

By (3), $\partial_{*}$ is one-one. Hence it remains to show that $H_{n}(\partial \tilde{X}) \rightarrow H_{n}(\tilde{X})$ is trivial, but this follows from (1) and the fact $H_{n}(\partial \tilde{X}) \cong \Lambda /(t-1)$. Q.E.D.

Finally we want to define the signature of a $(2 m-1)$-knot $K$ in $S^{2 m+1}$.

By Proposition 2.5(4), $\partial_{*}: H_{2 m}(\tilde{X}, \partial \tilde{X}) \rightarrow H_{2 m-1}(\partial \tilde{X})$ is an isomorphism which is compatible with $t_{*}$. On $\partial X$, we select an oriented $(2 m-1)$-sphere $S^{2 m-1}$ which is homologous to $K$ in $\mathrm{Cl}(U)$. If $m=1$, we further require that $S^{2 m-1}$ is a longitude of $\mathrm{Cl}(U)$. Let $e$ be the homology class in $H_{2 m-1}(\partial \tilde{X})$ represented by a lifting of $S^{2 m-1}$ to $\partial \tilde{X}$. Then $e$ generates $H_{2 m-1}(\partial \tilde{X})$ and is called the canonical generator of $H_{2 m-1}(\partial \tilde{X}) . e$ is uniquely determined by $K$ and satisfies $t_{*}(e)=e$. The element $\zeta=\partial_{*}^{-1}(e)$ of $H_{2 m}(\tilde{X}, \partial \tilde{X})$ will be called the fundamental class of $\tilde{X} . \zeta$ is uniquely determined by $K$ and satisfies $t_{*}(\zeta)=\zeta$.

By the universal-coefficient theorem for cohomology, we have

$$
H^{2 m}(\tilde{X}, \partial \tilde{X} ; Q) \cong \operatorname{Hom}\left(H_{2 m}(\tilde{X}, \partial \tilde{X} ; Q), Q\right), \quad u \mapsto\langle u,\rangle .
$$

Hence we can define two types of pairings $B_{1}$ and $B_{2}$ from $H^{m}(\tilde{X}, \partial \tilde{X} ; Q) \otimes$ $H^{m}(\tilde{X}, \partial \tilde{X} ; Q)$ to $Q$ by

$$
B_{1}(x, y)=\langle x \cup y, \zeta\rangle
$$


and

$$
B_{2}(x, y)=\left\langle x \cup t^{*} y+y \cup t^{*} x, \zeta\right\rangle
$$

for $x, y \in H^{m}(\tilde{X}, \partial \tilde{X} ; Q)$. By Lemma 4.4 of [2], we have

Proposition 2.6. $B_{1}$ is a dual pairing and $B_{1}(y, x)=(-1)^{m} B_{1}(x, y)$ for

$$
x, y \in H^{m}(\tilde{X}, \partial \tilde{X} ; Q) .
$$

For every positive integer $m, B_{2}$ is a quadratic form on the finite-dimensional vector space $H^{m}(\tilde{X}, \partial \tilde{X} ; Q)$. The signature of $B_{2}$ will be denoted by $\sigma_{2}(K)$.

If $m$ is even, $B_{1}$ is also a quadratic form on $H^{m}(\tilde{X}, \partial \tilde{X} ; Q)$ and the signature of $B_{1}$ will be denoted by $\sigma_{1}(K)$.

Theorem 5.2 of [2] shows that

Proposition 2.7. $\sigma_{1}(K)$ and $\sigma_{2}(K)$ are invariants of the $(2 m-1)$-knot type of $K$.

3. Proof of Theorem 1.1. Let $K, V, L$ and $\lambda$ be as in Theorem 1.1. Let $U$ be a tube of $L$ which is contained in Int $V$.

We will use the following notations:

$$
\begin{aligned}
X & =S^{n+2}-\text { Int } U, & & i_{1}: W \subset X, \\
W & =V-\text { Int } U, & & i_{2}: Y \subset X, \\
Y & =S^{n+2}-\text { Int } V, & & i_{3}: T \subset X, \\
T & =\text { Bdary } V . & &
\end{aligned}
$$

Choose a point $x \in T$. Let $\varphi$ be a homomorphism of $\pi_{1}(X, x)$ onto an infinite cyclic group $G=(t:)$ defined by

$$
\varphi(g)=t^{\operatorname{Link}(g, L)} \quad \text { for } g \in \pi_{1}(X, x),
$$

and $p: \tilde{X} \rightarrow X$ the infinite cyclic covering space belonging to $\operatorname{Ker}(\varphi)$. Put $\tilde{W}=$ $p^{-1}(W), \tilde{Y}=p^{-1}(Y)$ and $\tilde{T}=p^{-1}(T)$. Then $\tilde{X}=\tilde{W} \cup \tilde{Y}$ and $\tilde{T}=\tilde{W} \cap \tilde{Y}$.

First we will consider the case $\lambda \neq 0$. We want to show that if $\lambda \neq 0$ then

$$
\begin{aligned}
& \Delta_{L}^{q}(t)=\Delta_{K}^{q}\left(t^{\lambda}\right) \cdot \operatorname{order}_{\Gamma} H_{q}(\tilde{W} ; Q), \quad 1 \leqq q \leqq n-1, \\
& \left(t^{\lambda}-1\right) \cdot \Delta_{L}^{n}(t)=\Delta_{K}^{n}\left(t^{\lambda}\right) \cdot \operatorname{order}_{\Gamma} H_{n}(\tilde{W} ; Q) .
\end{aligned}
$$

Since $L \sim \lambda K$ in $V$, the homomorphism

is given by

$$
\pi_{1}(Y, x) \stackrel{i_{2 \#}}{\longrightarrow} \pi_{1}(X, x) \stackrel{\varphi}{\longrightarrow} G
$$

$$
\left(\varphi \cdot i_{2 \#}\right)(g)=t^{\lambda \cdot \operatorname{Link}(g, K)}
$$

for $g \in \pi_{1}(Y, x)$, and $\pi_{1}(Y, x)$ is mapped onto $H=\left(t^{\lambda}:\right)$ by $\varphi \cdot i_{2 \#}$. Let $Y_{1}$ be a pathcomponent of $\tilde{Y}$. Then, by Lemma $2.1, p \mid Y_{1}: Y_{1} \rightarrow Y$ is an infinite cyclic covering space of $Y$ belonging to $\operatorname{Ker}\left(\varphi \cdot i_{2 \#}\right) . Y$ is the complement of an open tube of $K$ in 
$S^{n+2}$ and $\varphi \cdot i_{2 \#}$ is the homomorphism given by (2.1) if we replace $t$ by $t^{\lambda}$ in (2.1). From these observations it follows that

$$
\operatorname{order}_{\Gamma^{\lambda}} H_{q}\left(Y_{1} ; Q\right)=\Delta_{K}^{q}\left(t^{\lambda}\right)
$$

Hence, by Corollary 2.2, we have

$$
\operatorname{order}_{\Gamma} H_{q}(\tilde{Y} ; Q)=\Delta_{K}^{q}\left(t^{\lambda}\right) \text {. }
$$

The homomorphism

$$
\pi_{1}(W, x) \stackrel{i_{1 \#}}{\longrightarrow} \pi_{1}(X, x) \stackrel{\varphi}{\longrightarrow} G
$$

is given by

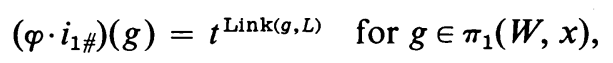

and is onto. Hence, by Lemma $2.1, p \mid \tilde{W}: \tilde{W} \rightarrow W$ is an infinite cyclic covering space belonging to $\operatorname{Ker}\left(\varphi \cdot i_{1 \#}\right)$.

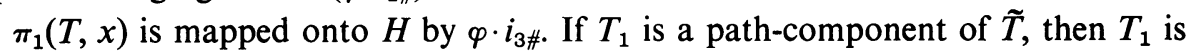
homeomorphic to $S^{n} \times R^{1}$ and

$$
\begin{aligned}
\operatorname{order}_{\Gamma^{\lambda}} H_{q}\left(T_{1} ; Q\right) & =t^{\lambda}-1, & & q=0, n, \\
& =1, & & \text { otherwise. }
\end{aligned}
$$

Hence, by Corollary 2.2, we have

$$
\begin{aligned}
\operatorname{order}_{\Gamma} H_{q}(\tilde{T} ; Q) & =t^{\lambda}-1, & & q=0, n, \\
& =1, & & \text { otherwise. }
\end{aligned}
$$

By using the Mayer-Vietoris sequence of $\tilde{W}$ and $\tilde{Y}$, (3.4) and Proposition 2.5(3), we can show that

$$
H_{q}(\tilde{X} ; Q) \cong{ }_{\Gamma} H_{q}(\tilde{W} ; Q) \oplus H_{q}(\tilde{Y} ; Q) \text { for } 1 \leqq q \leqq n-1,
$$

and

$$
0 \rightarrow H_{n}(\tilde{T} ; Q) \rightarrow H_{n}(\tilde{W} ; Q) \oplus H_{n}(\tilde{Y} ; Q) \rightarrow H_{n}(\tilde{X} ; Q) \rightarrow 0
$$

is exact.

By (3.3), it is clear that

$$
\Delta_{L}^{q}(t)=\Delta_{K}^{q}\left(t^{\lambda}\right) \cdot \operatorname{order}_{\Gamma} H_{q}(\tilde{W} ; Q) \text { for } 1 \leqq q \leqq n-1 .
$$

From (3.3), (3.4) and Assertion 1 of [3] it follows that

$$
\left(t^{\lambda}-1\right) \cdot \Delta_{L}^{n}(t)=\Delta_{K}^{n}\left(t^{\lambda}\right) \cdot \operatorname{order}_{\Gamma} H_{n}(\tilde{W} ; Q)
$$

Hence we have proved (3.1).

We now consider the case $\lambda=0$. By (3.2), the homomorphism

$$
\pi_{1}(Y, x) \stackrel{i_{2 \#}}{\longrightarrow} \pi_{1}(X, x) \stackrel{\varphi}{\longrightarrow} G
$$


is trivial. Since $Y$ has the homology of a circle, it follows from Corollary 2.3 that

$$
\begin{aligned}
H_{q}(\tilde{Y} ; Q) & \cong \Gamma, \quad q=0,1, \\
& \cong 0, \quad \text { otherwise }
\end{aligned}
$$

Similarly

$$
\pi_{1}(T, x) \stackrel{i_{3 \#}}{\longrightarrow} \pi_{1}(X, x) \stackrel{\varphi}{\longrightarrow} G
$$

is trivial and $H_{q}(\tilde{T} ; Q) \cong 0$ for $q \neq 0,1, n, n+1$. As before, $p \mid \tilde{W}: \tilde{W} \rightarrow W$ is an infinite cyclic covering space belonging to $\operatorname{Ker}\left(\varphi \cdot i_{1 \#}\right)$. Hence the Mayer-Vietoris sequence of $\tilde{W}$ and $\tilde{Y}$ implies that

$$
0 \rightarrow H_{1}(\tilde{T} ; Q) \rightarrow H_{1}(\tilde{W} ; Q) \oplus H_{1}(\tilde{Y} ; Q) \rightarrow H_{1}(\tilde{X} ; Q) \rightarrow 0
$$

is exact,

$$
H_{q}(\tilde{X} ; Q) \cong H_{q}(\tilde{W} ; Q) \text { for } q \neq 1, n
$$

and if $n \geqq 2$,

$$
0 \rightarrow H_{n}(\tilde{T} ; Q) \rightarrow H_{n}(\tilde{W} ; Q) \rightarrow H_{n}(\tilde{X} ; Q) \rightarrow 0
$$

is exact.

If $n \geqq 2, H_{1}(T) \rightarrow H_{1}(Y)$ is an isomorphism, and hence $H_{1}(\tilde{T} ; Q) \rightarrow H_{1}(\tilde{Y} ; Q)$ is a $\Gamma$-isomorphism. By using this fact and (3.5), one can show easily that

$$
H_{1}(\tilde{W} ; Q) \cong{ }_{\Gamma} H_{1}(\tilde{X} ; Q) \text {. }
$$

Therefore, if $n \geqq 2$,

$$
\Delta_{q}^{L}(t)=\operatorname{order}_{\Gamma} H_{q}(\tilde{W} ; Q), \quad 1 \leqq q \leqq n-1,
$$

and

$$
0 \rightarrow H_{n}(\tilde{T} ; Q) \rightarrow H_{n}(\tilde{W} ; Q) \rightarrow H_{n}(\tilde{X} ; Q) \rightarrow 0
$$

is exact.

If $n=1$, let $R$ and $S$ be a meridian and a longitude of $V$ respectively. $R$ represents a generator of $H_{1}(Y)$ and $S$ is a trivial element in $H_{1}(Y)$. Put $\widetilde{R}=p^{-1}(R)$ and $\tilde{S}=p^{-1}(S)$. Then it is easy to show that

$$
\begin{aligned}
& H_{1}(\tilde{T}) \cong H_{1}(\tilde{R}) \oplus H_{1}(\tilde{S}), \\
& H_{1}(\tilde{R}) \rightarrow H_{1}(\tilde{Y}) \text { is an isomorphism, and } \\
& H_{1}(\tilde{S}) \rightarrow H_{1}(\tilde{Y}) \text { is trivial. }
\end{aligned}
$$

From this fact and (3.5) it follows that

$$
0 \rightarrow H_{1}(\tilde{S} ; Q) \rightarrow H_{1}(\tilde{W} ; Q) \rightarrow H_{1}(\tilde{X} ; Q) \rightarrow 0
$$

is exact.

Now let $K^{\prime}, V^{\prime}, L^{\prime}$ and $\lambda$ be as in Theorem 1.1. The previous argument is also valid for this case. We will put a superscript "'", to the notations in the previous 
argument to denote the corresponding objects for the case $K^{\prime}, V^{\prime}, L^{\prime}$ and $\lambda$. We may assume that $f\left(U^{\prime}\right)=U$ and $f\left(x^{\prime}\right)=x$.

It is easy to show that $f_{\#}: \pi_{1}\left(W^{\prime}, x^{\prime}\right) \rightarrow \pi_{1}(W, x)$ is an isomorphism which makes the diagram

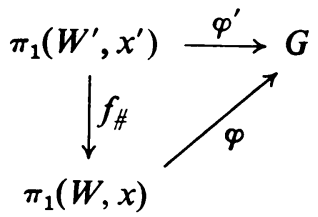

commutative. Hence $f$ induces an orientation-preserving homeomorphism

$$
\tilde{f}: \tilde{W}^{\prime} \rightarrow \tilde{W}
$$

which is compatible with $t$ and

$$
\tilde{f_{*}}: H_{q}\left(\tilde{W}^{\prime} ; Q\right) \cong{ }_{\Gamma} H_{q}(\tilde{W} ; Q)
$$

for every integer $q$.

Since $K^{\prime}$ is trivial, if $\lambda \neq 0$, we have

$$
\begin{aligned}
& \Delta_{L^{\prime}}^{q}(t)=\operatorname{order}_{\Gamma} H_{q}\left(\tilde{W}^{\prime} ; Q\right), \quad 1 \leqq q \leqq n-1, \\
& \left(t^{\lambda}-1\right) \cdot \Delta_{L^{\prime}}^{n}(t)=\operatorname{order}_{\Gamma} H_{n}\left(\tilde{W}^{\prime} ; Q\right) .
\end{aligned}
$$

Hence, if $\lambda \neq 0$, Theorem 1.1 follows from this fact, (3.1) and (3.11).

If $\lambda=0$ and $n \geqq 2$, by (3.8) and (3.11) we have $\Delta_{L^{\prime}}^{q}(t)=\Delta_{L}^{q}(t)$ for $1 \leqq q \leqq n-1$. Moreover, the diagram

$$
\begin{array}{ccc}
H_{n}\left(\tilde{T}^{\prime} ; Q\right) & \longrightarrow & H_{n}\left(\tilde{W}^{\prime} ; Q\right) \\
\tilde{f}_{*} \mid \cong \Gamma & & \cong \Gamma \downarrow \tilde{f}_{*}^{f} \\
H_{n}(\tilde{T} ; Q) & \longrightarrow & H_{n}(\tilde{W} ; Q)
\end{array}
$$

is commutative. Hence, by (3.9), there exists a $\Gamma$-isomorphism $H_{n}\left(\tilde{X}^{\prime} ; Q\right) \cong$ $H_{n}(\tilde{X} ; Q)$, which implies $\Delta_{L^{\prime}}^{n}(t)=\Delta_{L}^{n}(t)$.

If $\lambda=0$ and $n=1$, we may assume that $f\left(S^{\prime}\right)=S$. The diagram

$$
\begin{aligned}
& H_{1}\left(\tilde{S}^{\prime} ; Q\right) \longrightarrow H_{1}\left(\tilde{W}^{\prime} ; Q\right) \\
& \tilde{f}_{*} \downarrow \simeq \Gamma \quad \tilde{f}_{*} \preceq \Gamma \\
& H_{1}(\tilde{S} ; Q) \longrightarrow H_{1}(\tilde{W} ; Q)
\end{aligned}
$$

is commutative. Hence, by (3.10), we have $H_{1}\left(\tilde{X}^{\prime} ; Q\right) \cong{ }_{\Gamma} H_{1}(\tilde{X} ; Q)$, which yields $\Delta_{L^{\prime}}^{1}(t)=\Delta_{L}^{1}(t)$.

This completes the proof of Theorem 1.1.

REMARK. With slight modifications we can show that if $\lambda=0$, then $H_{q}\left(\tilde{X}^{\prime}\right)$ $\cong{ }_{\Lambda} H_{q}(\tilde{X})$ for every integer $q$. 
4. Proof of Theorem 1.2. Suppose that $n=2 m-1$. In the case $m=1$, Theorem 1.2 follows from Theorem 9 of [5] and the equivalence of Erle's definition and Trotter's [1]. Hence we will assume that $m \geqq 2$. We will use the same notations as in $\S 3$.

Let $K, V, L$ and $\lambda$ be as in Theorem 1.2. The Mayer-Vietoris sequence of $(\tilde{W}, \partial \tilde{W})$ and $(\tilde{Y}, \partial \tilde{Y})$ yields

$$
\begin{aligned}
& H_{q}(\tilde{X}, \partial \tilde{W}) \stackrel{\cong}{\leftrightarrows} H_{q}(\tilde{W}, \partial \tilde{W}) \oplus H_{q}(\tilde{Y}, \partial \tilde{Y}) \text { and } \\
& H^{q}(\tilde{X}, \partial \tilde{W}) \stackrel{\cong}{\longrightarrow} H^{q}(\tilde{W}, \partial \tilde{W}) \oplus H^{q}(\tilde{Y}, \partial \tilde{Y})
\end{aligned}
$$

for every integer $q$.

As in $\S 3$, we will divide our consideration into two cases. First we will consider the case $\lambda \neq 0$. By Lemma 2.1, $\tilde{Y}$ is the disjoint union of $Y_{1}, \ldots, Y_{\mu}$ such that

(1) $\mu=|\lambda|$,

(2) $p \mid Y_{i}: Y_{i} \rightarrow Y$ is an infinite cyclic covering space of $Y$ belonging to

$$
\operatorname{Ker}\left(\varphi \cdot i_{2 \#}\right) \text {, }
$$

where $\varphi \cdot i_{2 \#}$ is given by (3.2),

(3) $t$ carries $Y_{1}$ to $Y_{2}, Y_{2}$ to $Y_{3}, \ldots, Y_{\mu}$ to $Y_{1}$.

Note that $Y$ is the complement of an open tube of $K$ in $S^{2 m+1}$.

We denote the inclusion maps as follows:

$$
\begin{aligned}
j:(\tilde{X}, \partial \tilde{X}) & \subset(\tilde{X}, \partial \tilde{W}), \\
j_{0}:(\tilde{W}, \partial \tilde{W}) & \subset(\tilde{X}, \partial \tilde{W}), \\
j_{v}:\left(Y_{v}, \partial Y_{v}\right) & \subset(\tilde{X}, \partial \tilde{W}), \quad v=1,2, \ldots, \mu .
\end{aligned}
$$

Let $e \in H_{2 m-1}(\partial \tilde{X})$ be the canonical generator and $\zeta=\partial_{*}^{-1}(e)$ the fundamental class of $\tilde{X}$. For $\nu=1, \ldots, \mu$, let $e_{\nu} \in H_{2 m-1}\left(\partial Y_{v}\right)$ be the canonical generator and $\zeta_{v}=\partial_{v_{*}}^{-1}\left(e_{v}\right)$ the fundamental class of $Y_{v}$, where

$$
\partial_{v *}: H_{2 m}\left(Y_{v}, \partial Y_{v}\right) \stackrel{\cong}{\longrightarrow} H_{2 m-1}\left(\partial Y_{v}\right) .
$$

Since $t_{*}\left(e_{1}\right)=e_{2}, \ldots, t_{*}\left(e_{\mu}\right)=e_{1}$, we have

$$
t_{*}\left(\zeta_{1}\right)=\zeta_{2}, \quad t_{*}\left(\zeta_{2}\right)=\zeta_{3}, \quad \cdots, \quad t_{*}\left(\zeta_{\mu}\right)=\zeta_{1} .
$$

By Proposition $2.5(3), H_{2 m}(\tilde{X}) \cong 0$ and $H_{2 m}\left(Y_{v}\right) \cong 0$ for $\nu=1,2, \ldots, \mu$. Hence the Mayer-Vietoris sequence of $\tilde{W}$ and $\tilde{Y}$ yields $H_{2 m}(\tilde{W}) \cong 0$, which implies that $\partial_{0 *}: H_{2 m}(\tilde{W}, \partial \tilde{W}) \rightarrow H_{2 m-1}(\partial \tilde{W})$ is one-one.

We want to show that

(4.3) there exists a unique element $\zeta_{0} \in H_{2 m}(\tilde{W}, \partial \tilde{W})$ such that

$$
\partial_{0 *}\left(\zeta_{0}\right)=e-(\operatorname{sign} \lambda) \cdot\left(e_{1}+\cdots+e_{\mu}\right) .
$$


The isomorphism

$$
h_{*}: H_{2 m}(\tilde{W}, \partial \tilde{W}) \oplus H_{2 m}\left(Y_{1}, \partial Y_{1}\right) \oplus \cdots \oplus H_{2 m}\left(Y_{\mu}, \partial Y_{\mu}\right) \rightarrow H_{2 m}(\tilde{X}, \partial \tilde{W})
$$

is given by

$$
h_{*}\left(x_{0}, x_{1}, \ldots, x_{\mu}\right)=\sum_{v=0}^{\mu} j_{v_{*}}\left(x_{v}\right)
$$

for $x_{0} \in H_{2 m}(\tilde{W}, \partial \tilde{W})$ and $x_{v} \in H_{2 m}\left(Y_{v}, \partial Y_{v}\right), \nu=1,2, \ldots, \mu$. There exist a unique element $\zeta_{0} \in H_{2 m}(\tilde{W}, \partial \tilde{W})$ and unique integers $\varepsilon_{1}, \ldots, \varepsilon_{\mu}$ such that

$$
j_{*}(\zeta)=h_{*}\left(\zeta_{0}, \varepsilon_{1} \zeta_{1}, \ldots, \varepsilon_{\mu} \zeta_{\mu}\right)
$$

If we operate $t_{*}$ on (4.5), by (4.2) and the fact $t_{*}(\zeta)=\zeta$, we obtain

$$
j_{*}(\zeta)=h_{*}\left(t_{*}\left(\zeta_{0}\right), \varepsilon_{\mu} \zeta_{1}, \varepsilon_{1} \zeta_{2}, \ldots, \varepsilon_{\mu-1} \zeta_{\mu}\right)
$$

Since $h_{*}$ is one-one, we have $\varepsilon_{1}=\varepsilon_{2}=\cdots=\varepsilon_{\mu}$.

By the commutativity of the following diagrams

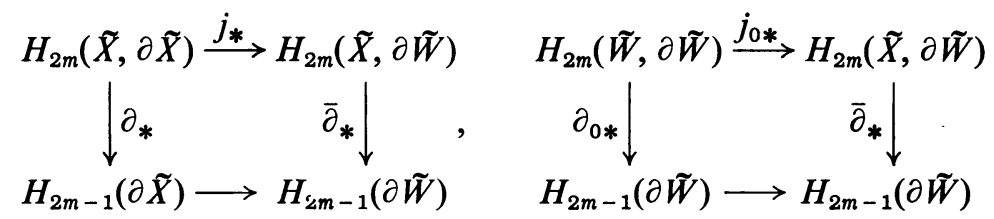

and

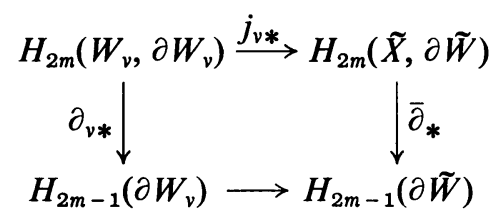

we obtain

$$
\begin{aligned}
\bar{\partial}_{* j} j_{*}(\zeta)=e, \quad \bar{\partial}_{*} h_{0 *}\left(\zeta_{0}\right)=\partial_{0 *}\left(\zeta_{0}\right), & \text { and } \bar{\partial}_{* j_{v *}}\left(\zeta_{v}\right)=e_{v}, \\
\nu & =1,2, \ldots \mu .
\end{aligned}
$$

Hence, by (4.4)-(4.6), we have $\partial_{0 *}\left(\zeta_{0}\right)=e-\varepsilon_{1}\left(e_{1}+\cdots+e_{\mu}\right)$ in $H_{2 m-1}(\partial \tilde{W})$.

To complete (4.3), we need only to show that $\varepsilon_{1}=\operatorname{sign} \lambda$. Since the diagram

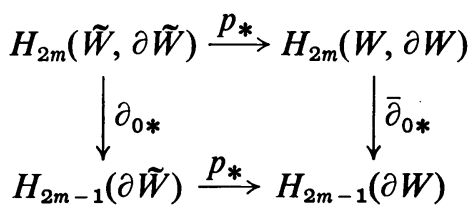

is commutative, in $H_{2 m-1}(\partial W)$ we have

$$
\bar{\partial}_{0 *}\left(p_{*}\left(\zeta_{0}\right)\right)=p_{*}\left(\partial_{0 *}\left(\zeta_{0}\right)\right)=p_{*}(e)-\varepsilon_{1}\left(p_{*}\left(e_{1}\right)+\cdots+p_{*}\left(e_{\mu}\right)\right) .
$$

Hence $p_{*}(e)=\varepsilon_{1}\left(p_{*}\left(e_{1}\right)+\cdots+p_{*}\left(e_{\mu}\right)\right)$ in $H_{2 m-1}(W)$, and in $H_{2 m-1}(V)$. This shows that $L \sim \varepsilon_{1} \mu K$ in $V$ and $\varepsilon_{1}=\operatorname{sign} \lambda$. This completes the proof of (4.3). 
Since $m \geqq 2, j^{*}: H^{m}(\tilde{X}, \partial \tilde{W}) \rightarrow H^{m}(\tilde{X}, \partial \tilde{X})$ is an isomorphism. The homomorphism

$$
h^{*}: H^{m}(X, \partial \tilde{W}) \rightarrow H^{m}(\tilde{W}, \partial \tilde{W}) \oplus H^{m}\left(Y_{1}, \partial Y_{1}\right) \oplus \cdots \oplus H^{m}\left(Y_{\mu}, \partial Y_{\mu}\right)
$$

given by

$$
h^{*}(x)=\left(j_{0}^{*}(x), j_{1}^{*}(x), \ldots, j_{\mu}^{*}(x)\right)
$$

for $x \in H^{m}(\tilde{X}, \partial \tilde{W})$ is an isomorphism. For an element $x$ of $H^{m}(\tilde{X}, \partial \tilde{X})$, we denote by $\bar{x}$ the image of $x$ under the isomorphism $h^{*} \cdot j^{*-1}$.

Let $x_{1}, \ldots, x_{r}$ and $y_{1}, \ldots, y_{s}$ be elements of $H^{m}(\tilde{X}, \partial \tilde{X} ; Q)$ such that

$\left\{\bar{x}_{1}, \ldots, \bar{x}_{r}\right\}$ is a basis for $H^{m}(\tilde{W}, \partial \tilde{W} ; Q)$ and

$\left\{\bar{y}_{1}, \ldots, \bar{y}_{s}\right\}$ is a basis for $H^{m}\left(Y_{\mu}, \partial Y_{\mu} ; Q\right)$.

Then $\left\{t^{* v} \bar{y}_{1}, \ldots, t^{* v} \bar{y}_{s}\right\}$ is a basis for $H^{m}\left(W_{\mu-v}, \partial W_{\mu-v} ; Q\right)$ for $\nu=0,1, \ldots, \mu-1$, and

$$
\mathscr{B}: x_{1}, \ldots, x_{r} ; y_{1}, \ldots, y_{s} ; \ldots ; t^{* \mu-1} y_{1}, \ldots, t^{* \mu-1} y_{s}
$$

forms a basis for $H^{m}(\tilde{X}, \partial \tilde{X} ; Q)$.

First we want to consider the matrix $A$ representing the pairing $B_{1}$ with respect to the basis $\mathscr{B}$. By making use of the fact that $j_{*}(\zeta)=h_{*}\left(\zeta_{0}, \varepsilon \zeta_{1}, \ldots, \varepsilon \zeta_{\mu}\right)$, where $\varepsilon=\operatorname{sign} \lambda$, we can show that

$$
\begin{aligned}
B_{1}\left(x_{i}, x_{j}\right) & =\left\langle\bar{x}_{i} \cup \bar{x}_{j}, \zeta_{0}\right\rangle, & & \\
B_{1}\left(t^{* v} y_{i}, t^{* v} y_{j}\right) & =\varepsilon\left\langle\bar{y}_{i} \cup \bar{y}_{j}, \zeta_{\mu}\right\rangle, & & 0 \leqq \nu \leqq \mu-1, \\
B_{1}\left(x_{i}, t^{* v} y_{j}\right) & =B_{1}\left(t^{* v} y_{i}, x_{j}\right)=0, & & 0 \leqq \nu \leqq \mu-1, \\
B_{1}\left(t^{* v} y_{i}, t^{* v} y_{j}\right) & =0, & & \nu \neq \tau .
\end{aligned}
$$

Let

$$
C=\left\|\left\langle\bar{x}_{i} \cup \bar{x}_{j}, \zeta_{0}\right\rangle\right\|_{1 \leqq i, j \leqq r}
$$

and

$$
D=\left\|\varepsilon\left\langle\bar{y}_{i} \cup \bar{y}_{j}, \zeta_{\mu}\right\rangle\right\|_{1 \leqq i, j \leqq s}
$$

Then we have

$$
A=\left(\begin{array}{lll}
C & & 0 \\
& D & D_{D}^{\mu} \\
0 & & \cdot
\end{array}\right] .
$$

If $m$ is even, then

$$
\text { signature }\left\|\left\langle\bar{y}_{i} \cup \bar{y}_{j}, \zeta_{\mu}\right\rangle\right\|_{1 \leqq i, j \leqq s}=\sigma_{1}(K)
$$

and

$$
\sigma_{1}(L)=\text { signature } C+\varepsilon \mu \sigma_{1}(K)=\text { signature } C+\lambda \sigma_{1}(K) .
$$


We now want to consider the matrix $B$ representing $B_{2}$ with respect to $\mathscr{B}$. We can show that

$$
\begin{aligned}
& B_{2}\left(x_{i}, x_{j}\right)=\left\langle\bar{x}_{i} \cup t^{*} \bar{x}_{j}+\bar{x}_{j} \cup t^{*} \bar{x}_{i}, \zeta_{0}\right\rangle, \\
& B_{2}\left(t^{* v} y_{i}, t^{* v+1} y_{j}\right)=\varepsilon\left\langle\bar{y}_{j} \cup \bar{y}_{i}, \zeta_{\mu}\right\rangle \text {, } \\
& B_{2}\left(y_{i}, t^{* \mu-1} y_{j}\right)=\varepsilon\left\langle\bar{y}_{i} \cup t^{*} \bar{y}_{j}+\bar{y}_{j} \cup t^{*} \bar{y}_{i}, \zeta_{1}\right\rangle \text { if } \mu=1 \text {, } \\
& =\varepsilon\left\langle y_{i} \cup t^{* \mu} y_{j}, \zeta_{\mu}\right\rangle \quad \text { if } \mu>1, \\
& B_{2}\left(t^{* v} y_{i}, t^{* \tau} y_{j}\right)=0 \text { if } \mu>1 \text { and }|\nu-\tau| \neq 1, \mu-1 \text {, } \\
& B_{2}\left(x_{i}, t^{* v} y_{j}\right)=0 \text {. }
\end{aligned}
$$

Let

and

$$
E=\left\|\left\langle\bar{x}_{i} \cup t^{*} \bar{x}_{j}+\bar{x}_{j} \cup t^{*} \bar{x}_{i}, \zeta_{0}\right\rangle\right\|_{1 \leqq i, j \leqq r}
$$

$$
F=\left\|\varepsilon\left\langle\bar{y}_{i} \cup t^{* \mu} \bar{y}_{j}, \zeta_{\mu}\right\rangle\right\|_{1 \leqq i, j \leqq s}
$$

Then we have

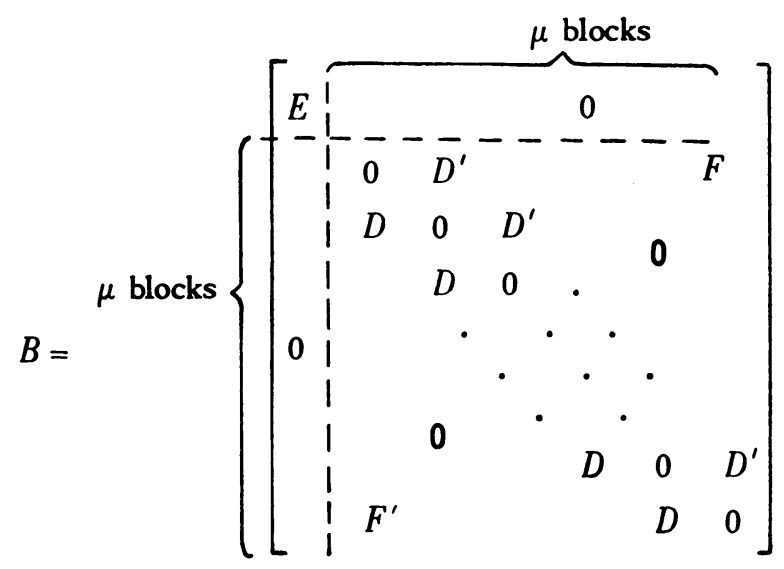

If $\mu$ is even, it is easy to show that the matrix

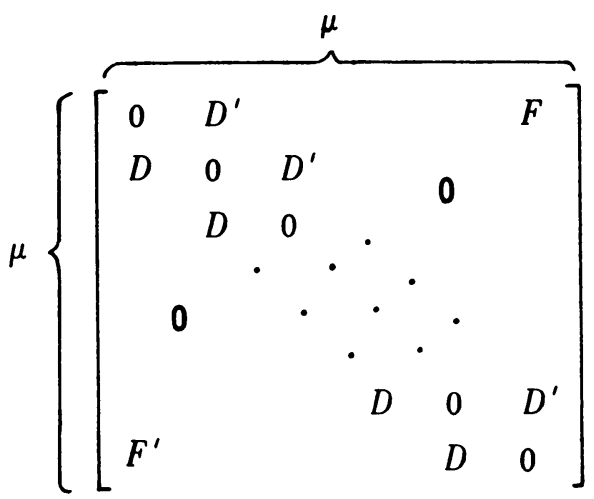

is congruent to its negative and its signature is zero. Hence if $\mu$ is even,

$$
\sigma_{2}(L)=\text { signature } E \text {. }
$$


Proposition 2.6 implies that $D^{\prime}=(-1)^{m} D$ and $D$ is nonsingular. If $\mu$ is odd, by adding $(-1)^{m+1}$ times the first row block to the third and $(-1)^{m+1}$ times the first column block to the third; $(-1)^{m+1}$ times the new third row block to the 5 th and $(-1)^{m+1}$ times the new third column block to the 5 th $; \ldots ;(-1)^{m+1}$ times the new $(\mu-2)$ th row block to the $\mu$ th and $(-1)^{m+1}$ times the new $(\mu-2)$ th column block to the $\mu$ th, we can show that (4.8) is congruent to the matrix

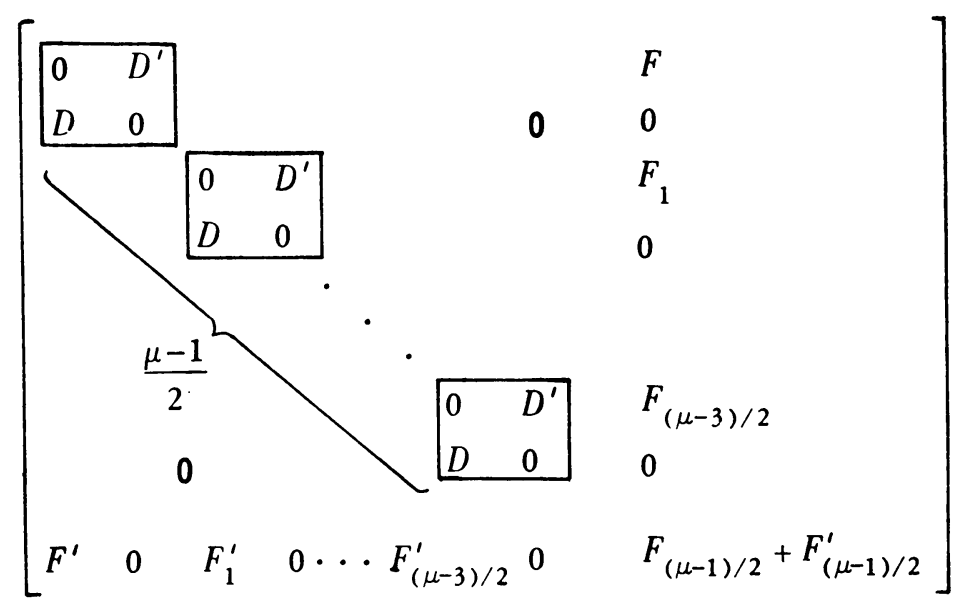

where $F_{i}=(-1)^{(m+1) i} F, i=1, \ldots,(\mu-1) / 2$. Since $D$ is nonsingular, (4.10) is congruent to

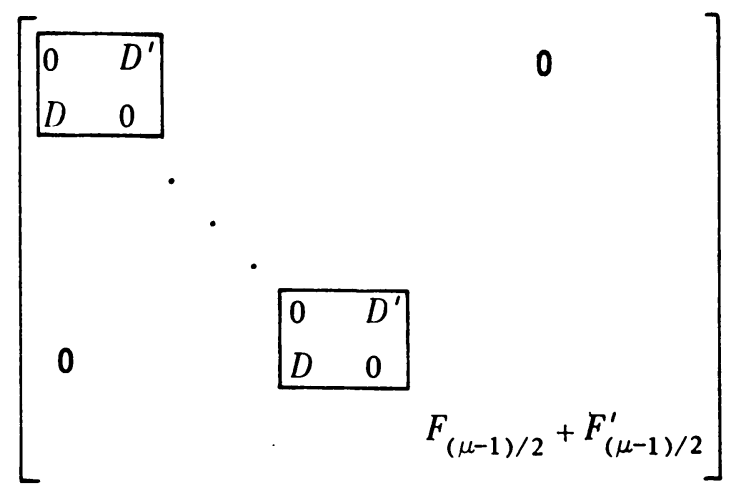

It is clear that signature $\left[\begin{array}{ll}0 & D^{\prime} \\ D & 0\end{array}\right]=0$. One can show that

$$
\begin{aligned}
F_{(\mu-1) / 2}+F_{i \mu-1) / 2}^{\prime} & =(-1)^{(m+1) \cdot(\mu-1) / 2}\left\|\varepsilon\left\langle\bar{y}_{i} \cup t^{* \mu} \bar{y}_{j}+\bar{y}_{j} \cup t^{* \mu} \bar{y}_{i}, \zeta_{\mu}\right\rangle\right\|_{1 \leqq i, j \leqq s} \\
& =(-1)^{(m+1) \cdot(\lambda-1) / 2}\left\|\left\langle\bar{y}_{i} \cup t^{* \lambda} \bar{y}_{j}+\bar{y}_{j} \cup t^{* \lambda} \bar{y}_{i}, \zeta_{\mu}\right\rangle\right\|_{1 \leqq i, j \leqq s .} .
\end{aligned}
$$

Therefore, if $\mu$ is odd,

$$
\sigma_{2}(L)=\text { signature } E+(-1)^{(m+1) \cdot(\lambda-1) / 2} \sigma_{2}(K) .
$$

Now, as in $\S 3, f^{*}: H^{m}(\tilde{W}, \partial \tilde{W}) \rightarrow H^{m}\left(\tilde{W}^{\prime}, \partial \tilde{W}^{\prime}\right)$ is an isomorphism which is compatible with $t^{*}$ and $\tilde{f}_{*}: H_{2 m}\left(\tilde{W}^{\prime}, \partial \tilde{W}^{\prime}\right) \rightarrow H_{2 m}(\tilde{W}, \partial \tilde{W})$ is an isomorphism such 
that the diagram

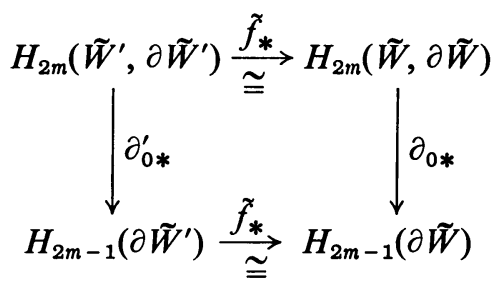

is commutative. Since $\tilde{f}_{*}\left(e^{\prime}\right)=e$ and $\tilde{f}_{*}\left(e_{1}^{\prime}+\cdots+e_{\mu}^{\prime}\right)=e_{1}+\cdots+e_{\mu}$,

$$
\begin{aligned}
\partial_{0 *}\left(\tilde{f}_{*}\left(\zeta_{0}^{\prime}\right)\right) & =\tilde{f}_{*}\left(\partial_{0 *}^{\prime}\left(\zeta_{0}^{\prime}\right)\right)=\tilde{f}_{*}\left(e^{\prime}-(\operatorname{sign} \lambda) \cdot\left(e_{1}^{\prime}+\cdots+e_{\mu}^{\prime}\right)\right) \\
& =e-(\operatorname{sign} \lambda) \cdot\left(e_{1}+\cdots+e_{\mu}\right) .
\end{aligned}
$$

Hence, by (4.3), we have $\tilde{f}_{*}\left(\zeta_{0}^{\prime}\right)=\zeta_{0}$.

$\left\{\tilde{f}^{*}\left(\bar{x}_{1}\right), \ldots, \tilde{f}^{*}\left(\bar{x}_{r}\right)\right\}$ is a basis for $H^{m}\left(\tilde{W}^{\prime}, \partial \tilde{W}^{\prime} ; Q\right)$ which satisfies

$$
\left\langle\tilde{f}^{*}\left(\bar{x}_{i}\right) \cup \tilde{f}^{*}\left(\bar{x}_{j}\right), \zeta_{0}^{\prime}\right\rangle=\left\langle\bar{x}_{i} \cup \bar{x}_{j}, \zeta_{0}\right\rangle
$$

and

$$
\left\langle\tilde{f}^{*}\left(\bar{x}_{i}\right) \cup t^{*} \tilde{f}^{*}\left(\bar{x}_{j}\right)+\tilde{f}^{*}\left(\bar{x}_{j}\right) \cup t^{*} \tilde{f}^{*}\left(\bar{x}_{i}\right), \zeta_{0}^{\prime}\right\rangle=\left\langle\bar{x}_{i} \cup t^{*} \bar{x}_{j}+\bar{x}_{j} \cup t^{*} \bar{x}_{i}, \zeta_{0}\right\rangle .
$$

Since $K^{\prime}$ is trivial, by (4.7), (4.9) and (4.11), we have

$$
\sigma_{1}\left(L^{\prime}\right)=\text { signature }\left\|\left\langle\tilde{f}^{*}\left(\bar{x}_{i}\right) \cup \tilde{f}^{*}\left(\bar{x}_{j}\right), \zeta_{0}^{\prime}\right\rangle\right\|=\text { signature } C
$$

and

$$
\sigma_{2}\left(L^{\prime}\right)=\text { signature }\left\|\left\langle\tilde{f}^{*}\left(\bar{x}_{i}\right) \cup t^{*} \tilde{f}^{*}\left(\bar{x}_{j}\right)+\tilde{f}^{*}\left(\bar{x}_{j}\right) \cup t^{*} \tilde{f}^{*}\left(\bar{x}_{i}\right), \zeta_{0}^{\prime}\right\rangle\right\|=\text { signature } E \text {. }
$$

Therefore we have shown that

$$
\begin{aligned}
\sigma_{2}(L) & =\sigma_{2}\left(L^{\prime}\right) & & \text { when } \lambda \text { is even, } \\
& =\sigma_{2}\left(L^{\prime}\right)+(-1)^{(m+1) \cdot(\lambda-1) / 2} \sigma_{2}(K) & & \text { when } \lambda \text { is odd, }
\end{aligned}
$$

and if $m$ is even,

$$
\sigma_{1}(L)=\sigma_{1}\left(L^{\prime}\right)+\lambda \sigma_{1}(K) .
$$

This completes the case $\lambda \neq 0$.

Finally we want to consider the case $\lambda=0 . \quad \tilde{Y}$ consists of countably many copies of $Y$ and $\partial \tilde{Y}$ consists of countably many copies of $\partial Y \cong S^{2 m-1} \times S^{1}$. In the Mayer-Vietoris sequence of $(\tilde{Y}, \varnothing)$ and $(\tilde{W}, \partial \tilde{X})$, we can show that

$$
H^{m}(\tilde{X}, \partial \tilde{X}) \stackrel{h^{*}}{\longrightarrow} H^{m}(\tilde{W}, \partial \tilde{X})
$$

is an isomorphism and

$$
0 \longrightarrow H_{2 m}(\partial \tilde{Y}) \longrightarrow H_{2 m}(\tilde{W}, \partial \tilde{X}) \stackrel{h_{*}}{\longrightarrow} H_{2 m}(\tilde{X}, \partial \tilde{X}) \longrightarrow 0
$$

is exact. Note that $m \geqq 2$. Hence there exists an element $\zeta_{0} \in H_{2 m}(\tilde{W}, \partial \tilde{X})$ such that $h_{*}\left(\zeta_{0}\right)=\zeta$. 
Let $\left\{x_{1}, \ldots, x_{r}\right\}$ be a basis for $H^{m}(\tilde{X}, \partial \tilde{X} ; Q)$. Then $\left\{\bar{x}_{1}, \ldots, \bar{x}_{r}\right\}$ is a basis for $H^{m}(\tilde{W}, \partial \tilde{X} ; Q)$, where $\bar{x}_{i}=h^{*}\left(x_{i}\right)$ for $i=1, \ldots, r$, and

$$
\begin{aligned}
& B_{1}\left(x_{i}, x_{j}\right)=\left\langle\bar{x}_{i} \cup \bar{x}_{j}, \zeta_{0}\right\rangle, \\
& B_{2}\left(x_{i}, x_{j}\right)=\left\langle\bar{x}_{i} \cup t^{*} \bar{x}_{j}+\bar{x}_{j} \cup t^{*} \bar{x}_{i}, \zeta_{0}\right\rangle .
\end{aligned}
$$

As before, $\tilde{f}^{*}: H^{m}(\tilde{W}, \partial \tilde{X}) \rightarrow H^{m}\left(\tilde{W}^{\prime}, \partial \tilde{X}^{\prime}\right)$ and $\tilde{f}_{*}: H_{2 m}\left(\tilde{W}^{\prime}, \partial \tilde{X}^{\prime}\right) \rightarrow H_{2 m}(\tilde{W}, \partial \tilde{X})$ are isomorphisms and $\tilde{f}_{*}\left(e^{\prime}\right)=e$. Since the diagram

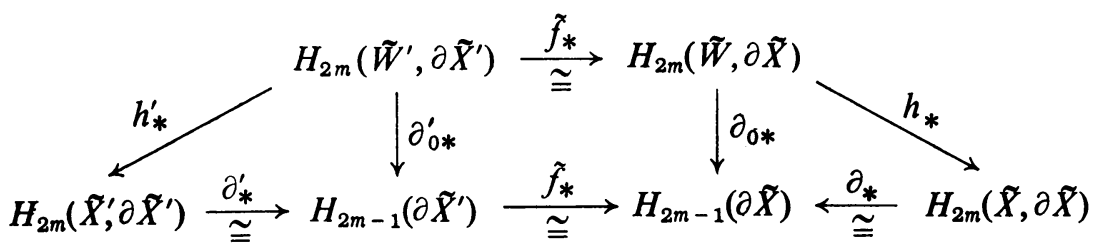

is commutative, $\zeta_{0}^{\prime}=\tilde{f}_{*}^{-1}\left(\zeta_{0}\right) \in H_{2 m}\left(\tilde{W}^{\prime}, \partial \tilde{X}^{\prime}\right)$ satisfies $h_{*}^{\prime}\left(\zeta_{0}^{\prime}\right)=\zeta^{\prime}$.

$\left\{\tilde{f}^{*}\left(\bar{x}_{1}\right), \ldots, \tilde{f}^{*}\left(\bar{x}_{r}\right)\right\}$ is a basis for $H^{m}\left(\tilde{W}^{\prime}, \partial \tilde{X}^{\prime} ; Q\right)$ and $\left\{x_{1}^{\prime}, \ldots, x_{r}^{\prime}\right\}$ is a basis for $H^{m}\left(\tilde{X}^{\prime}, \partial \tilde{X}^{\prime} ; Q\right)$, where $x_{i}^{\prime}=h^{*-1}\left(\tilde{f}^{*}\left(\bar{x}_{i}\right)\right)$ for $i=1, \ldots, r$. Hence

$$
\begin{aligned}
B_{1}\left(x_{i}^{\prime}, x_{j}^{\prime}\right) & =\left\langle\bar{f}^{*}\left(\bar{x}_{i}\right) \cup \bar{f}^{*}\left(\bar{x}_{j}\right), \zeta_{0}^{\prime}\right\rangle \\
& =\left\langle\bar{x}_{i} \cup \bar{x}_{j}, \zeta_{0}\right\rangle=B_{1}\left(x_{i}, x_{j}\right) .
\end{aligned}
$$

Likewise we have $B_{2}\left(x_{i}^{\prime}, x_{j}^{\prime}\right)=B_{2}\left(x_{i}, x_{j}\right)$. Therefore we have shown that $\sigma_{2}\left(L^{\prime}\right)=\sigma_{2}(L)$ and, if $m$ is even, $\sigma_{1}\left(L^{\prime}\right)=\sigma_{1}(L)$. This completes the proof of Theorem 1.2.

\section{REFERENCES}

1. D. Erle, Die quadratische Form eines Knotens und ein Satz über Knotenmannigfaltigkeiten, J. Reine Angew. Math. 236 (1969), 174-218. MR 40 \#2106.

2. - Quadratische Formen als Invarianten von Einbettungen der Kodimension 2, Topology 8 (1969), 99-114. MR 38 \#6576.

3. J. W. Milnor, Infinite cyclic coverings, Conference on the Topology of Manifolds (Michigan State University, E. Lansing, Mich., 1967), Prindle, Weber and Schmidt, Boston, Mass., 1968, pp. 115-133. MR 39 \#3497.

4. H. Seifert, On the homology invariants of knots, Quart J. Math. Oxford Ser. (2) 1 (1950), 23-32. MR 11, 735.

5. Y. Shinohara, On the signature of knots and links, Trans. Amer. Math. Soc. 156 (1971), 273285.

6. H. F. Trotter, Homology of group systems with applications to knot theory, Ann. of Math. (2) 76 (1962), 464-498. MR 26 \#761.

University of Toronto, Toronto, CANAdA 